\title{
Modelo para especificar, ejecutar y controlar la impermeabilización de cubiertas planas en concreto reforzado construidas en la ciudad de Bogotá.
}

\author{
C. Chinome ${ }^{1 *}$, C. Rios-Fresneda ${ }^{2}$ \\ *Autor de Contacto: cchinome@unal.edu.co \\ ${ }^{1}$ Estudiante maestría en construcción, facultad de artes, Universidad Nacional de Colombia, Bogotá, Colombia \\ ${ }^{2}$ Profesor Universidad Nacional de Colombia, Grupo de investigación de estructuras, facultad de ingeniería, \\ Universidad Nacional de Colombia, Bogotá, Colombia
}

\begin{abstract}
RESUMEN
La investigación realizada tiene como objeto evaluar un modelo diseñado para optimizar la impermeabilización de cubiertas de tipo plana en concreto, con conocimiento de las características de las tecnologías disponibles y unifique como solución el criterio de reducción de la permeabilidad del concreto y la aplicación del recubrimiento impermeable como un solo sistema que trabaje en conjunto. El modelo establece un compendio de criterios como una "guía paso a paso" que será de gran utilidad para los constructores. Su diseño metodológico se basa en la investigación proyectual, modelos sistémicos y la experiencia del ejercicio profesional de los autores. Se concluye que el modelo propuesto permitirá que la cubierta de tipo plana no cumpla su vida útil antes del tiempo esperado.
\end{abstract}

Palabras clave: Impermeabilización, concreto, recubrimiento, especificación, control.

\section{RESUMO}

O objetivo da investigação realizada é avaliar um modelo desenhado para otimizar a impermeabilização de coberturas planas em concreto, com conhecimento das características das tecnologias disponíveis e unificar como solução o critério de redução da permeabilidade do concreto e a aplicação do revestimento impermeável, como um sistema único que funciona em conjunto. O modelo estabelece um compêndio de critérios como um "guia passo a passo" que será de grande utilidade para os construtores. Seu desenho metodológico é baseado na pesquisa de projetos, modelos sistêmicos e na experiência da prática profissional dos autores. Conclui-se que o modelo proposto permitirá que a cobertura do tipo plano excede a expectativa de vida esperada. Palavras-chave: Impermeabilização, concreto, revestimento, especificação, controle

\begin{abstract}
The purpose of the research carried out is to evaluate a model designed to optimize the waterproofing of flat roofs in concrete, with knowledge of the characteristics of the available technologies and to unify as a solution the criterion of permeability reduction of concrete and the application of the waterproof coating as a single system that works together. The model establishes a compendium of criteria as a "step-by-step" guide that will be of great use to builders. Its methodological design is based on project research, systemic models, and the experience of the authors' professional practice. It is concluded that the proposed model will allow the flat roof to
\end{abstract}


exceed the expected useful life.

Keywords: Waterproofing, concrete, coating, specification, control.

\section{INTRODUCCIÓN}

Las cubiertas planas en concreto reforzado son construidas como cerramiento de la parte superior del edificio y en su geometría no se contemplan pendientes superiores al 5\%. Las cubiertas deben garantizar un nivel de servicio adecuado para la exposición al medio ambiente que le rodea, los niveles de cargas de diseño y a los cambios climáticos que se presentan (Monjo \& Maldonado, 2001; Gonçalves et al., 2019).

Estos elementos estructurales de la edificación son, desde el punto de vista ingenieril, encargados de transmitir fuerza a los elementos verticales del sistema estructural. Su diseño debe soportar su propio peso, cargas por elementos no estructurales de acabados, cargas vivas, cargas por empozamiento de agua, cargas por granizo, vibraciones de equipos y las acciones dinámicas derivadas por un sismo. Además de un correcto diseño estructural, la cubierta debe considerar el correcto diseño de la mezcla de concreto, tendiendo en cuenta el grado de exposición del medio ambiente y cumpliendo los requisitos de durabilidad necesarios para su correcto funcionamiento, (AIS 100, 2010).

Las edificaciones desde el punto de vista arquitectónico están compuestas por un sistema que contribuye a mediar entre lo inestable de por fuera y lo estable de adentro del edificio; este sistema se conoce como envolvente. La envolvente se compone de todas las uniones de muros exteriores, cubierta, aleros o voladizos, cimentación, vanos, aberturas acristaladas y sistemas de drenaje (Fernández, 2006). La cubierta en concreto reforzado es un elemento que hace parte de la envolvente del edificio (Pedrosa \& Del Río, 2018); por tal motivo es necesario no solo tener un criterio estructural en su diseño, sino también la visión del confort que debe generar la cubierta a sus usuarios.

Las cubiertas en concreto reforzado son un hito para el proyecto de construcción, que en la práctica se suele subestimar y se ejecuta sin revisar el proceso constructivo en sus diferentes etapas. Por ejemplo, una mala práctica en el proceso de impermeabilización en la cubierta traerá como consecuencia lesiones físicas, la aparición de filtraciones y el acortamiento de la vida útil.

Las filtraciones son un fenómeno que suele ser visto en distintas perspectivas. El cliente o usuario observa la filtración como una "gotera" que afecta su comodidad y puede dañar sus muebles. El constructor lo ve como una garantía que debe soportar con el fin de evitar la ejecución de una póliza de estabilidad. El aplicador de impermeabilizantes lo ve como una postventa que debe solucionar para poder cobrar su retención de dinero por garantía. Un especificador experimentado ve el fenómeno como una lesión física que genera lesiones mecánicas y lesiones químicas en la estructura de concreto y que trae como principal consecuencia la afectación de la estabilidad y reducción de la vida útil de la cubierta y como posteriormente la afectación de la comodidad y confort del usuario.

Cuando la cubierta es el objeto de estudio de una investigación y esta hace parte de la envolvente de la edificación, necesariamente las lesiones dentro de ella harán parte de los principales propósitos de una auscultación, y por ello las filtraciones en cubiertas de concreto reforzado se definen como un tipo de lesión física que se manifiesta porque el agua atraviesa la sección transversal antes de ser evacuada por el sistema de drenaje. El agua puede entrar y atravesar las cubiertas de concreto por sus intersticios, al tener alta permeabilidad en la superficie porosa o por lesiones mecánicas como grietas y fisuras que permiten el paso de este líquido. 
Una clasificación inicial de las filtraciones (Carretero, et al., 2017) se presenta de este modo:

- Filtración localizada: Penetración de agua a través de puntos débiles de un elemento constructivo, de forma aislada (situada en puntos específicos).

- Humedad por filtración: Entrada y / o presencia de agua por puntos débiles de una construcción elemento, de forma generalizada (situado en superficies más extensas).

La condición de diseño deseada para garantizar que la cubierta funcione correctamente es la estanqueidad, o capacidad de impermeabilización ante la lluvia (Carretero \& de Brito, 2016). Si la cubierta no cuenta con esta condición, el agua accede a través de la sección transversal, dando paso a potenciales lesiones que inician las filtraciones. Para evitar que esta situación suceda es importante contar con una combinación de tecnologías dispuestas para lograr una impermeabilización óptima, las cuales se deben especificar y presupuestar antes de iniciar un proyecto de construcción (Rodríguez, et al., 2004).

La prevención de las filtraciones suele ser subestimada. Una vez ocurren propietario y constructor suelen no dimensionar el conjunto de consideraciones técnicas y económicas que se deben tener para solucionar el problema. Las tecnologías disponibles para prevenir y corregir las filtraciones se componen de un conjunto de criterios establecidos para cada etapa de la impermeabilización e interrumpir el paso del agua. Enfatizar en el estándar mínimo necesario para la calidad del proceso constructivo de impermeabilización, es la principal motivación para emprender la investigación presentada y brindar así un aporte al conocimiento.

\section{ESTRUCTURA EN LA TIPOLOGÍA DEL MODELO PROPUESTO}

Las cubiertas bien especificadas deben cumplir como mínimo con un recubrimiento impermeable. Sin embargo, para las solicitaciones climáticas de algunas ciudades (v.gr. Bogotá) no es suficiente contemplar con un mero recubrimiento y se requiere del apoyo de otros sistemas de impermeabilización adicionales. La propuesta de del modelo propuesto se puede observar en la figura 1.

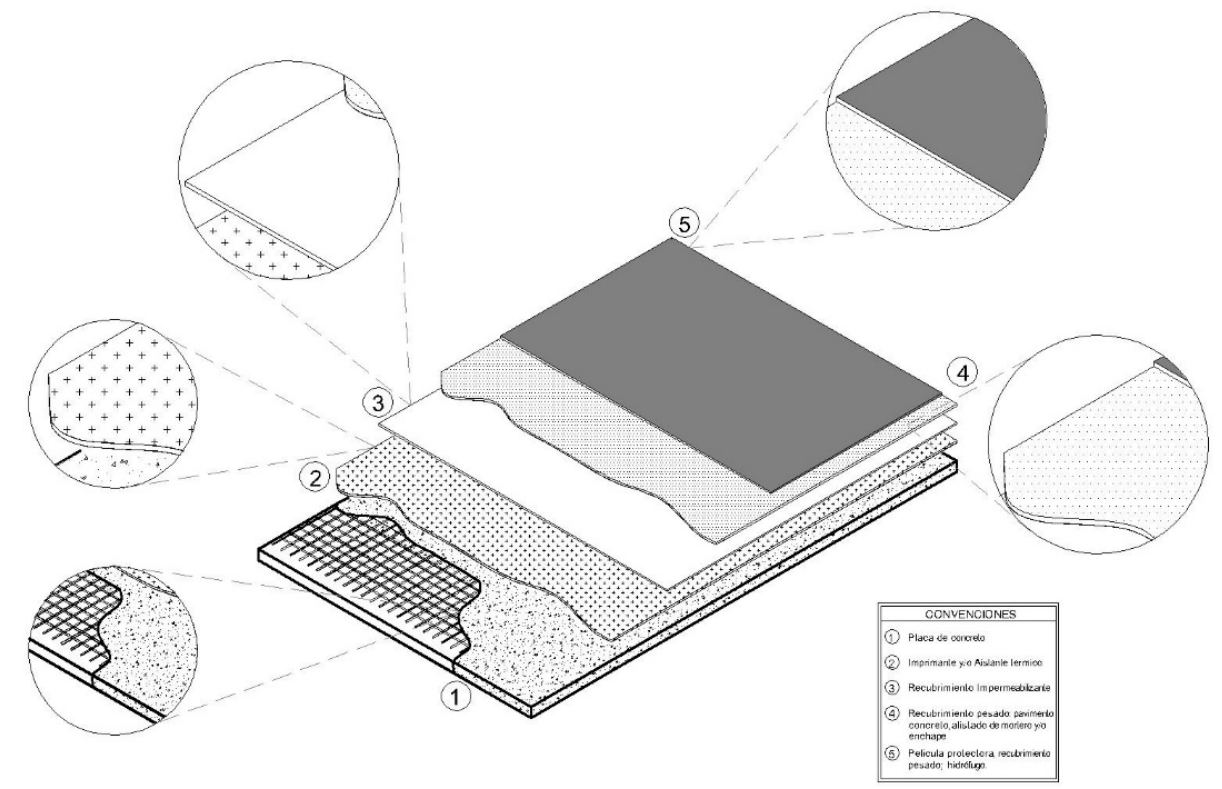

Figura 1: Estructura en la tipología de impermeabilización de cubiertas planas en concreto reforzado del modelo (elaboración Propia) 


\section{PROCEDIMIENTO}

\subsection{La investigación proyectual y los modelos sistémicos.}

Los proyectos de construcción inicialmente se desarrollaron mediante un pensamiento sistémico que obedece un orden lógico de las cosas. Ese orden se establece mediante unas normas de construcción prescriptivas (García, 2014), para convertir su idealización en una materialización por medio de un proceso constructivo (Monjo, 2005). Este pensamiento se viene implementando desde que se creó la teoría general de los sistemas por Von Bertalanffy (Hernández, 2008; Villate \& Tamayo, 2010).

La arquitectura como disciplina ha estado en constante evolución y desarrollo de metodologías para proyectar construcciones en sus diferentes etapas: Diseño, ejecución, control, validación y mantenimiento. Los arquitectos en la realización de proyectos no solo ven el proyectar como una secuencia lógica que se desarrolla mediante un pensamiento mecánico con una serie de pasos con el fin de solucionar un problema, también se interpreta como un pensamiento subjetivo en el que el profesional realiza un especulativo sobre el objeto de estudio y materializa una idea por medio de materiales y procesos constructivos. Esta metodología se conoce como investigación proyectual (Martínez, 2013; Alba, 2016; Salas, 2017; Godinho \& Verde, 2017).

El modelo propuesto tiene como fundamento dos corrientes de pensamientos en el desarrollo de proyectos, combinada con la experiencia profesional adquirida en la práctica profesional del autor y las cuales se proponen aquí como lecciones aprendidas (Navarrete, 2016).

\subsection{Etapas consideradas en el modelo}

La investigación desarrollada propone un modelo de impermeabilización integrado con las siguientes fases: diseño, recomendaciones del proceso constructivo, criterios de aceptación, recomendaciones de mantenimiento y otras opciones para optimizar el proceso de impermeabilización. Las diferentes etapas consideradas se pueden visualizar en la Figura 2.

\subsubsection{Diseño}

Los sistemas de impermeabilización para cubiertas elaboradas con concreto reforzado requieren de un diseño previo a su intervención. El diseñador de los sistemas de impermeabilización se le conoce como especificador. A partir del "lápiz del especificador" comienza la planeación del sistema de impermeabilización. Un especificador es un profesional de la ingeniería civil, arquitectura o construcción con experiencia en materiales y en aplicaciones, que tiene la responsabilidad de definir la materialidad y secuencia del proceso constructivo del sistema de impermeabilización (Racusin,1989).

Al culminar el proceso de diseño se debe el entregable es un plano de intervenciones con: dimensiones de los elementos, tipo de material con normativa aplicable para cada especificación, un documento que contiene el presupuesto y una memoria técnica del proceso constructivo, con las respectivas recomendaciones para la intervención (García, 2017; Racusin,1989).

\subsubsection{Funcionalidad}

Al considerar la funcionalidad de la cubierta se recomienda tener en cuenta los siguientes aspectos: Las condiciones del medio ambiente donde se emplazará el recubrimiento de la cubierta. Existen notorios efectos del cambio climático en varias ciudades del mundo.. Como ejemplo de lo anterior se pueden citar las lluvias con granizo que Bogotá experimento entre 2019 y 2020 . La obstrucción de drenajes, y los daños parciales en las cubiertas se convirtieron en los orígenes de filtraciones 
no previstas.(Peña \& Pabón, 2020). Los recubrimientos de la estructura de concreto reforzado y los sistemas de drenaje deberán soportar entonces solicitaciones unas mayores exigencias de precipitaciones y granizadas (Ruggiero \&Rutila, 1990).

Un segundo aspecto para considerar por quien especifica es el tipo de tráfico que debe tolerar la cubierta:. trafico peatonal permanente u ocasional o tráfico vehicular. También se debe considerar si el diseño es para obra nueva o para una intervención preventiva o correctiva. (Ruggiero \& Rutila; Racusin; Wohl \& LaFraugh 1990).

Una solicitación tan exigente como las anteriores corresponde a los efectos de los asentamientos diferenciales sobre la cubierta. Este fenómeno puede causar agrietamientos y fisuras en las cubiertas. Su aparición puede aducir un gran número de razones: Suelos expansivos, diseños geotécnicos insuficientes, están dentro de las más comunes. Suele citarse la expansión como el término que describe los cambios volumétricos del suelo donde se emplaza la estructura de cimentación. Sin embargo, el efecto en cualquier caso es el mismo: daño estructural, sin que la cubierta escape a ello. Cuando se evidencia que la edificación tiene diferenciales en sus ejes de apoyo y estos no alcanzan a ser resistidos uniformemente por la estructura, surge la necesidad de, emplear recubrimientos no adheridos a la placa. De lo contrario se habrá especificado equívocamente (Ruggiero \& Rutila, 1990).

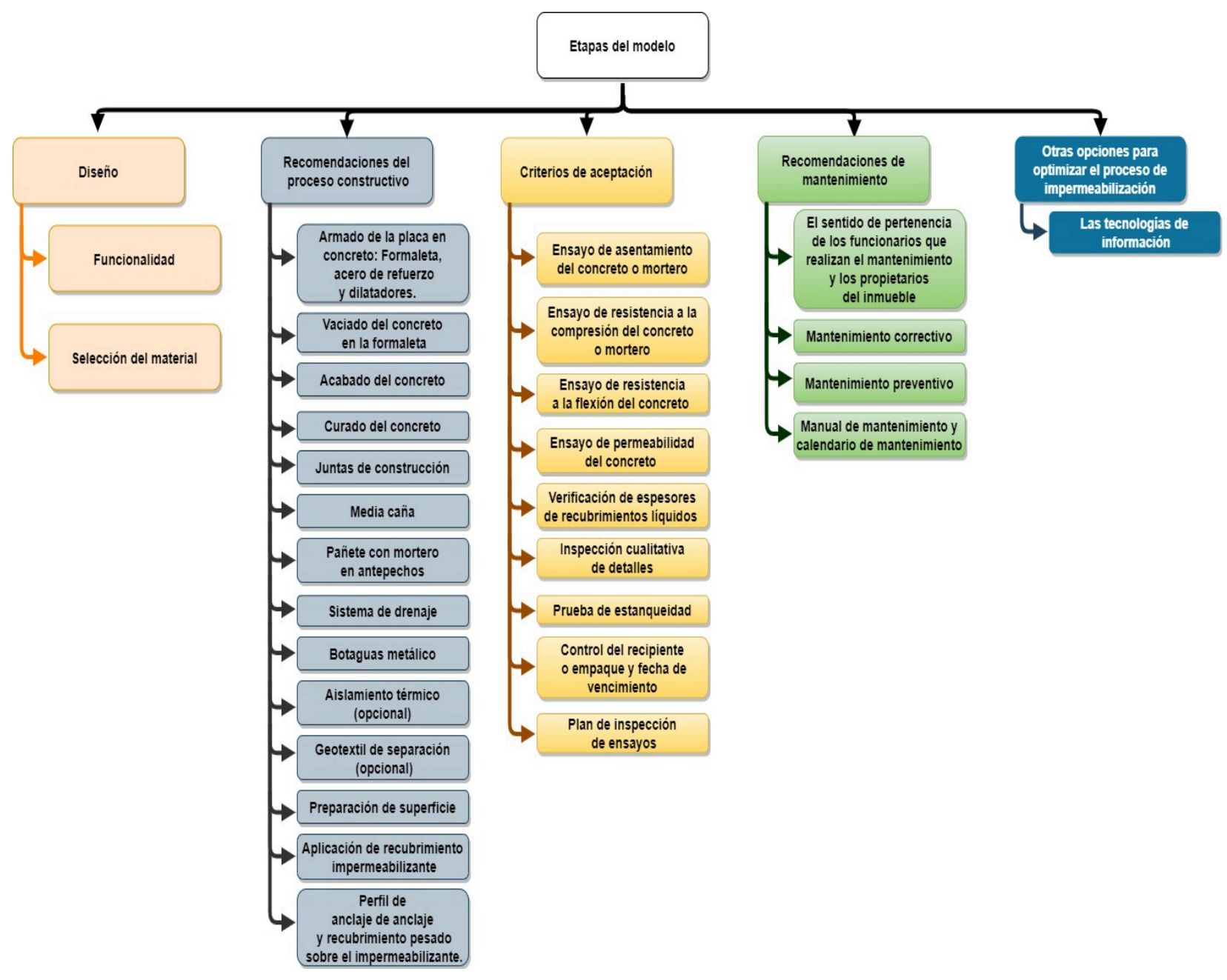

Figura 2: Etapas consideradas en el modelo (elaboración Propia) 


\subsubsection{Materialidad}

Las cubiertas también requieren un especial detallado en la selección de materiales. Estos deben cumplir las condiciones de calidad suficientes para cumplir el propósito para el cual se va a emplear (Arredondo; Ruggiero \& Rutila; Racusin, 1990). Algunas recomendaciones para tener en cuenta en el modelo propuesto son:

El concreto que constituya la cubierta debe reducir su permeabilidad con el fin de tener una estructura que sea durable. Para lograr que el concreto reduzca su permeabilidad se puede considerar: aumentar la resistencia a la compresión del concreto (Comité ACI 201, 2012; Montejo, et al.,2013; Matallana 2019), utilizar aditivos y/o materiales cementicios complementarios en la mezcla, o realizar un cuidadoso proceso de curado Comité ACI 201; Matallana, 2019; Pillai, et al.,2020).

El reducir la permeabilidad del concreto se puede considerar como un sistema colaborante esencial, que puede complementarse con el uso de un recubrimiento adicional en la parte superior de la sección de la cubierta. El uso de recubrimientos para protección de superficies en concreto es también una alternativa para alargar la vida útil de estructuras expuestas, disminuyendo el riesgo ante la penetración de agentes agresivos por difusión, migración o absorción capilar (Medeiros et al., 2015).

Algunos autores (Lesur, 2010) clasifican los sistemas de acuerdo con la familia de materiales a la que pertenecen, según su lugar de aplicación y según su temperatura de aplicación. A partir de esta clasificación, se establece una nueva manera de ver los sistemas de recubrimiento sobre el concreto, de la siguiente forma: adheridos, no adheridos y sistemas electrónicos de detección de fugas, como se puede observar en la figura 3.

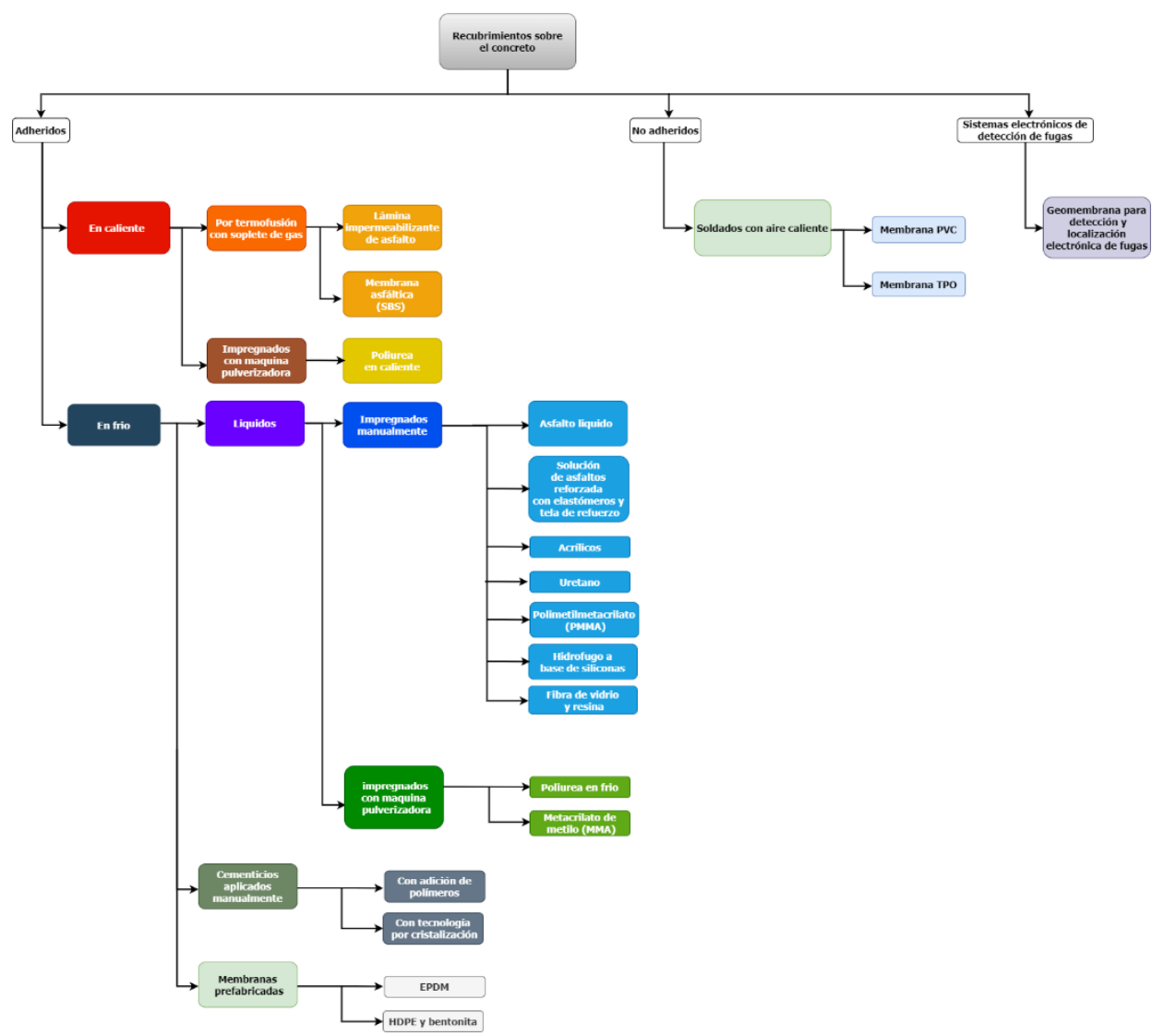

Figura 3: Clasificación de los recubrimientos sobre el concreto (elaboración Propia) 


\subsection{Recomendaciones del proceso constructivo.}

El proceso constructivo es el conjunto de reglas que se deben seguir de forma prescriptiva, con el fin de materializar elementos que conforman un sistema. Este a su vez hace parte del programa arquitectónico de la edificación (Fernández, 2006; García, 2014; Monjo, 2005). El modelo propuesto tiene como salida de la etapa de diseño los planos de detalle, el presupuesto y la memoria descriptiva de las especificaciones; los cuales, a su vez se convierten en el parámetro de entrada para la etapa del proceso constructivo del sistema. Se recomienda rigurosidad en su cumplimiento, con el fin de que el sistema de impermeabilización que se construye sea congruente con el diseño. Antes de iniciar la construcción, es recomendable revisar con el equipo de trabajo los detalles constructivos de los planos, la memoria descriptiva y las fichas técnicas de los materiales especificados. Adicionalmente, es importante conciliar una explicación en una instancia preoperacional por parte del especificador y de un asesor del fabricante de las tecnologías a emplear. Esto va a generar una adecuada transferencia de tecnología, garantizando un entendimiento previo del sistema constructivo que se va a ejecutar (Racusin, 1989).

\subsection{Criterios de aceptación}

Existen varias opciones para definir un estándar de calidad mínimo. Un ejemplo internacionalmente aceptado y que permite la construcción de un conjunto de criterios mínimos dentro de un sistema de ejecución es la ISO 9001:2015: Sistemas de gestión de la calidad. Cada País tiene una estructura para certificar sistemas y dentro de ellos pueden existir los de las empresas de construcción o supervisión que contemplen listas de chequeo para recibir la construcción de un sistema de impermeabilización. Una organización (v.gr. propietarios, constructores o supervisores) podrá construir una serie de criterios de aceptación y corresponderán al conjunto de parámetros y condiciones deseables que deben ser cumplidos para dar por aceptada la entrega de un producto o servicio, (PMI, 2017). Todo servicio que se preste en un área específica debe llevar un proceso de seguimiento, medición, análisis y evaluación, con el fin de verificar que el proceso realizado cumpla los parámetros preestablecidos. Para aplicar mecanismos de control de calidad en el modelo propuesto el principio de control de variables, control de atributos y no-control (Cortés,2017).

El control de variables consiste en la aceptación o rechazo de un producto a través de la medición de un parámetro de gran importancia, (Cortés,2017). En el modelo propuesto se establecen en esta clasificación la inspección el ensayo de asentamiento del concreto o mortero, el ensayo de resistencia la compresión del concreto o mortero, el ensayo de resistencia a flexión del concreto y la verificación de espesores en recubrimientos líquidos, son buena muestra de ello.

El control de atributos consiste en una clasificación de aceptación o rechazo de un producto por un parámetro de observación cualitativa y no por una medición, (Cortés,2017). En el modelo propuesto se establecen en esta clasificación la inspección cualitativa de detalles y la prueba de estanqueidad.

El no-control de atributos consiste en todo aquello que no se controla directamente por la organización. Por ejemplo, un producto que viene certificado por el fabricante, (Cortés,2017). En el modelo propuesto se aplica no-control para los aditivos, adiciones e impermeabilizantes. En ellos solo se verifica la etiqueta de sello del fabricante, integridad del recipiente o empaque y la fecha de vencimiento.

En los proyectos se debe realizar una planeación de ensayos y criterios de aceptación necesarios para aprobar el servicio o producto, esto se conoce como plan de inspección de ensayos, (AIS 100, 2010; PMI, 2017). En los sistemas de impermeabilización en los numerales anteriores se desarrollaron una serie de criterios de aceptación basados en la normativa legal vigente y experiencia profesional de autor. 
El realizar un plan de inspección de ensayos en un proceso de impermeabilización de cubiertas tiene como fin, el verificar la calidad del sistema, (Alba, et al., 2013). Los parámetros del sistema se verifican desde: La materialidad del concreto desde el punto de vista físico y mecánico, la aplicación del recubrimiento, el aspecto cualitativo de los procesos constructivos y la integridad de los productos previo a su aplicación. En la Tabla 1. se presenta la propuesta de plan de inspección de ensayo recomendada para un proceso de impermeabilización de cubiertas planas.

Tabla 1. Plan de inspección de ensayos para el proceso de impermeabilización de cubiertas planas

\begin{tabular}{|c|c|c|c|c|}
\hline $\begin{array}{l}\text { Nombre del } \\
\text { ensayo }\end{array}$ & $\begin{array}{l}\text { Tipo de } \\
\text { ensayo }\end{array}$ & Criterio de aceptación & $\begin{array}{c}\text { Responsable } \\
\text { de realizar } \\
\text { el ensayo } \\
\end{array}$ & $\begin{array}{c}\text { Responsable } \\
\text { de verificar } \\
\text { los resultados }\end{array}$ \\
\hline $\begin{array}{l}\text { Asentamiento del } \\
\text { concreto } \\
\text { (ICONTEC, NTC } \\
396 ; 2017 \text { ) }\end{array}$ & $\begin{array}{c}\text { No } \\
\text { destructivo }\end{array}$ & $\begin{array}{c}2.5 \mathrm{~cm} \text { y } 7,5 \mathrm{~cm} \text { en } \\
\text { concretos convencionales. } \\
455 \mathrm{~mm} \text { y } 810 \mathrm{~mm} \text { de } \\
\text { extensión de la mezcla en } \\
\text { estado fresco/ Entre } 2 \\
\text { segundos y } 10 \text { segundos } \\
\text { para extenderse } 500 \mathrm{~mm} \text {, } \\
\text { para concretos } \\
\text { autocompactantes. } \\
\text { (Matallana, 2019) }\end{array}$ & Constructor & Interventor \\
\hline $\begin{array}{c}\text { Resistencia a la } \\
\text { compresión del } \\
\text { concreto NTC } 673 \\
\text { (ICONTEC, 2010) }\end{array}$ & Destructivo & $\begin{array}{c}\text { Mínimo } 17 \text { Mpa. Debe } \\
\text { coincidir con los diseños } \\
\text { establecidos por el } \\
\text { especificador y/o } \\
\text { diseñador estructural, } \\
\text { (AIS 100,2010) }\end{array}$ & $\begin{array}{c}\text { Laboratorio } \\
\text { especializado }\end{array}$ & Interventor \\
\hline $\begin{array}{c}\text { Flexión del } \\
\text { concreto NTC } \\
2871 \text { (ICONTEC, } \\
\text { 2018; INVIAS } \\
\text { 2013) }\end{array}$ & Destructivo & $\begin{array}{l}\text { Debe coincidir con los } \\
\text { diseños establecidos por } \\
\text { el Ing. Civil especialista } \\
\text { en ingeniería de } \\
\text { pavimentos (Matallana, } \\
\text { 2019; Montejo, Montejo } \\
\text { \& Montejo, 2013) }\end{array}$ & $\begin{array}{c}\text { Laboratorio } \\
\text { especializado }\end{array}$ & Interventor \\
\hline $\begin{array}{l}\text { Permeabilidad del } \\
\text { concreto NTC } \\
4483 \text { (ICONTEC, } \\
1998 \text { ) }\end{array}$ & Destructivo & $\begin{array}{l}\text { Penetración del agua } \\
\text { menor a } 30 \mathrm{~mm} \\
\text { (Matallana, 2019) }\end{array}$ & $\begin{array}{c}\text { Laboratorio } \\
\text { especializado }\end{array}$ & Interventor \\
\hline $\begin{array}{l}\text { Verificación de } \\
\text { espesores de } \\
\text { recubrimientos } \\
\text { líquidos } \\
\end{array}$ & $\begin{array}{c}\text { No } \\
\text { destructivo }\end{array}$ & $\begin{array}{c}\text { Debe coincidir con lo } \\
\text { establecido por el } \\
\text { fabricante y/o } \\
\text { especificador }\end{array}$ & Constructor & Interventor \\
\hline $\begin{array}{c}\text { Inspección } \\
\text { cualitativa de } \\
\text { detalles } \\
\end{array}$ & $\begin{array}{c}\text { No } \\
\text { destructivo }\end{array}$ & $\begin{array}{l}\text { Se rechaza en los } \\
\text { siguientes casos: }\end{array}$ & Constructor & Interventor \\
\hline
\end{tabular}




\begin{tabular}{|c|c|c|c|c|}
\hline & & $\begin{array}{c}\text { Grietas y fisuras en } \\
\text { elementos de concreto o } \\
\text { mortero. } \\
\text { Delaminación del } \\
\text { concreto. } \\
\text { Pozos de agua en la } \\
\text { cubierta de concreto. } \\
\text { agrietamiento o } \\
\text { desprendimiento de } \\
\text { imprimante o de } \\
\text { recubrimiento } \\
\text { impermeable. } \\
\text { Perdida de adherencia en } \\
\text { unión en los traslapos de } \\
\text { mantos o membranas } \\
\text { (Carretero et al., 2017; } \\
\text { Monjo \& Maldonado, } \\
\text { 2001) }\end{array}$ & & \\
\hline $\begin{array}{c}\text { Prueba de } \\
\text { estanqueidad }\end{array}$ & $\begin{array}{c}\text { No } \\
\text { destructivo }\end{array}$ & $\begin{array}{l}\text { No se deben presentar } \\
\text { filtraciones en un periodo } \\
\text { de } 24 \text { horas debajo de la } \\
\text { cubierta (Osuna, 2016) }\end{array}$ & Constructor & Interventor \\
\hline $\begin{array}{l}\text { Control del } \\
\text { recipiente o } \\
\text { empaque y fecha } \\
\text { de vencimiento }\end{array}$ & $\begin{array}{c}\text { No } \\
\text { destructivo }\end{array}$ & $\begin{array}{c}\text { No debe estar caducado } \\
\text { con respecto a la fecha } \\
\text { actual }\end{array}$ & Constructor & Interventor \\
\hline
\end{tabular}

\subsection{RECOMENDACIONES DE MANTENIMIENTO}

Antiguamente la gestión de proyectos se enfocaba en su desarrollo por etapas: Planear, organizar, ejecutar y controlar. Sin embargo, con el tiempo se fue necesario aclarar que se requieren servicios de mantenimiento o después de la entrega del proyecto. Estas postventas y mantenimientos preventivos o correctivos comenzaron a formar parte del ciclo de vida de los proyectos. Por esa razón, la metodología de dirección del proyecto se considera el mantenimiento como una filosofía que hace parte del proceso y son tenidos en cuenta incluso desde la etapa documental y contractual, (Consorcio RehabiMed, 2007; PMI, 2017).

En el caso específico de las cubiertas en concreto reforzado, debemos tomar en cuenta que su estructura y el recubrimiento impermeable estarán expuestas a la intemperie. Por esa razón es importante considerar un proceso de mantenimiento de los elementos que la conforman con el fin de prolongar su vida útil, (Monjo, 1986). Es importante que el mantenimiento inicie según lo especificado y desde el mismo día que se pone en funcionamiento la edificación En los edificios no se debiese llegar a la rehabilitación precipitadamente pues la vida útil se prolonga cuando se realizan los mantenimientos requeridos oportunamente. (Consorcio RehabiMed, 2007). 


\subsubsection{El sentido de pertenencia de los funcionarios que realizan el mantenimiento y los propietarios del inmueble.}

Es un aspecto filosófico que las personas no valoren las cosas, hasta no involucrarse directamente para lograrlas. El mantenimiento del inmueble debería ser una labor que involucre a la comunidad u organización. En algunas ocasiones no se presta la importancia necesaria a este aspecto y ocurre el deterioro o abandono del inmueble. Es importante se involucre a la comunidad con una apertura de la cubierta en donde se exponga el antes, durante y después del proyecto. También es recomendable capacitar al personal de mantenimiento con el fin de realizar las tareas fijas que garanticen la conservación del inmueble, (Consorcio RehabiMed, 2007).

\subsubsection{Mantenimiento Correctivo}

El mantenimiento correctivo es el que se asocia a la reparación que se realizan de forma inmediata de las fallas que ocurren por la degradación del sistema. Esto puede ocurre cuando se declara abandono y no se efectuaron con rutinas de mantenimiento preventivo, (Rodríguez,et al.,2004). Sin embargo, estos mantenimientos pueden suscitarse también por casos fortuitos en los que la naturaleza supera todas las consideraciones técnicas en una obra civil y se debe analizar si es posible realizar correcciones de forma puntual.

Los mantenimientos correctivos van a ocurrir en algún momento de la vida útil de la edificación. Es una realidad que si se realizan las rutinas de mantenimiento preventivo de forma rutinaria su frecuencia disminuirá, (Consorcio RehabiMed, 2007).

Existen algunos casos en los que el sistema no se interviene definitivamente y se realizan labores paliativas. Las labores paliativas son realizadas con el fin de realizar correcciones temporales de la falla, para que el sistema siga funcionando mientras se soluciona la causa raíz. En las cubiertas de concreto reforzado comúnmente se pueden realizar mecanismos de solución paliativas tales como: el uso de bandejas metálicas que recolecten las filtraciones, la aplicación de morteros de ultra fraguado con recubrimiento cementicio impermeable que obturan las fugas de forma provisional. En la figura 4 se puede observar las bandejas metálicas para recolectar el agua proveniente por filtración.

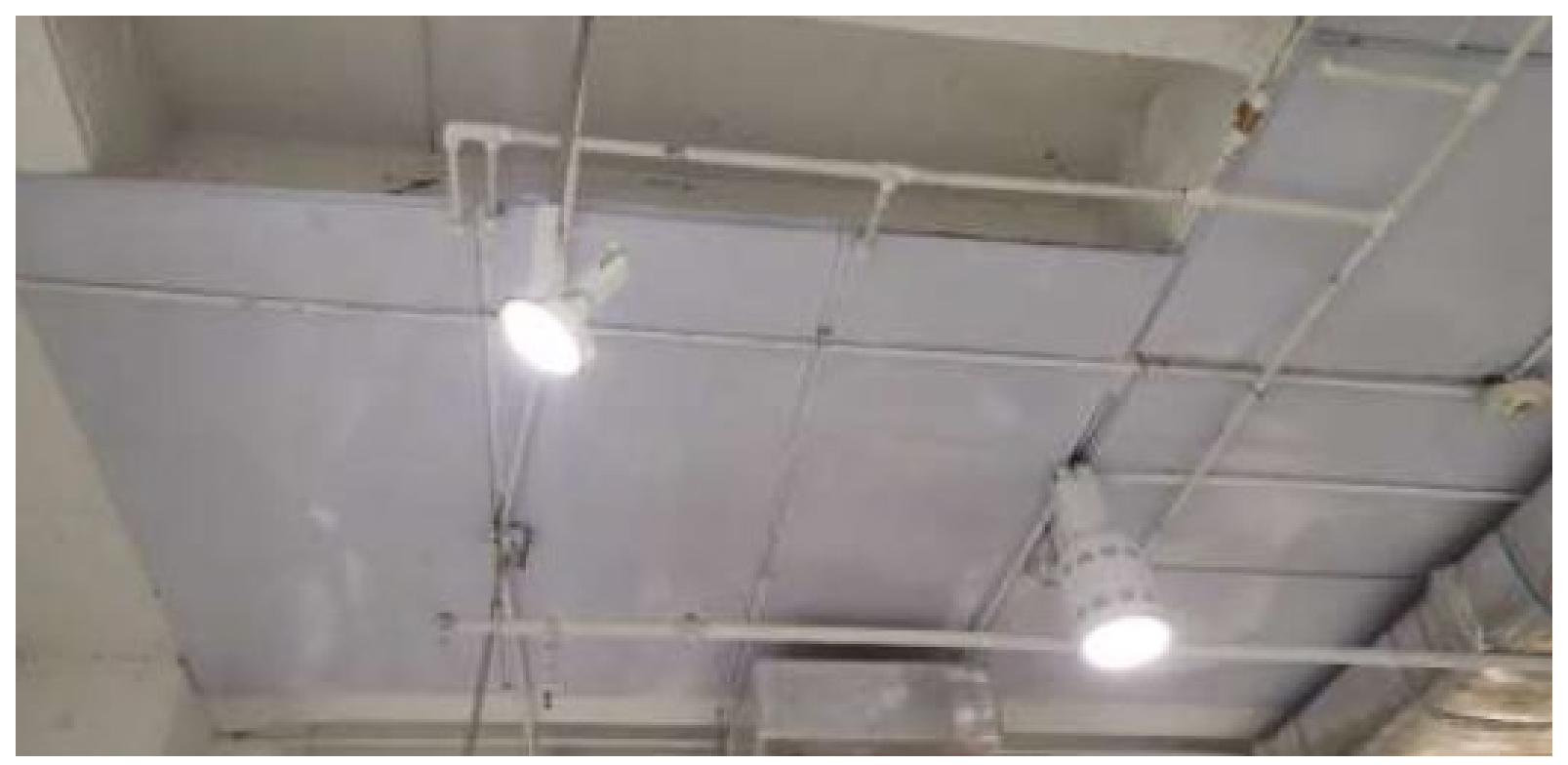

Figura 4: Bandejas metálicas para recolección de agua proveniente por filtración 


\subsubsection{Mantenimiento Preventivo.}

Las labores preventivas son todas aquellas labores rutinarias que se realizan de forma planeada y bajo una frecuencia, con el fin de conservar el inmueble en condiciones de mantenimiento optimas, (González, et al., 2020). También el mantenimiento puede ser visto como el conjunto de tareas que se realizan para prologar la vida útil del inmueble y alejarlo de la rehabilitación temprana, (Herrera, et al., 2016)Para realizar un mantenimiento preventivo en cubiertas de concreto reforzado se debe realizar unas rutinas que se dividen de la siguiente forma:

- Limpieza de sifones y canales: Todas las cubiertas tienen el propósito de proteger a la edificación desde la parte superior y permitir la habitabilidad en el inmueble, (Monjo, 2005).

- Las cubiertas planas deben evacuar el agua a los drenajes y para garantizar eso se debe evitar obstrucciones de sifones y canales. Es una tarea necesaria en todos los casos el realizar una limpieza superficial de hojas y material granular que pueda estar sobre ella y evitar que ocurra una obstrucción de drenajes.

- Revisión de materialidad y funcionamiento recomendado por el fabricante: Los sistemas de impermeabilización de cubiertas de acuerdo con su forma de aplicación y material requieren un mantenimiento preventivo distinto. Esto se debe consultar en la ficha técnica del fabricante e incluirse en el plan de mantenimiento.

\subsubsection{Manual De Mantenimiento Y Calendario De Mantenimiento.}

El manual de mantenimiento es una guía paso a paso de las labores preventivas y correctivas que se deben realizar al entregar el inmueble y es un documento entregable del proyecto. Su elaboración requiere de la consideración de tareas fijas necesarias para garantizar el correcto funcionamiento del inmueble, (Consorcio RehabiMed, 2007).

Bajo el parámetro de manual de mantenimiento es que el profesional de mantenimiento designado por el cliente y/o el personal técnico del cliente, realizan el calendario de mantenimiento. El calendario de mantenimiento son los días en que se establecen las rutinas fijas para garantizar las tareas preventivas para alargar la vida útil del inmueble, (Consorcio RehabiMed, 2007).

\section{DISCUSIÓN}

El modelo propuesto es un compendio de criterios y conocimientos resultantes de una revisión temática y de la experiencia profesional, que se estructuró con la secuencia de proyecto de construcción, con el fin de realizar un aporte a la academia dando una nueva perspectiva a los procesos constructivos en la impermeabilización de cubiertas planas en concreto.

El modelo propuesto ya se implementó en un proyecto profesional de uno de los autores y fue un caso de éxito. El proyecto donde se implementó es la cubierta de un centro comercial y se ejecutó entre mayo y octubre del 2020. El proceso constructivo desarrollado se ilustra por medio de un flujograma en la figura 5.

En la implementación del modelo se realizó prueba de estanqueidad al recubrimiento impermeable, la cual se puede observar en la figura 6.

Posteriormente se construye un recubrimiento pesado sobre la impermeabilización con mortero 1:3 mejorado con aditivo látex y juntas de dilatación con sello elastómero para controlar cambios volumétricos por temperatura. 
El recubrimiento pesado se puede observar en la figura 7. Finalmente, se aplicó un recubrimiento de protección para el recubrimiento pesado utilizando manto multicapa fabricado con base en asfaltos no oxidados modificados, con refuerzo central de fibra de vidrio y con acabado en "foil" de aluminio gofrado. El recubrimiento de protección se puede observar en la figura 8.

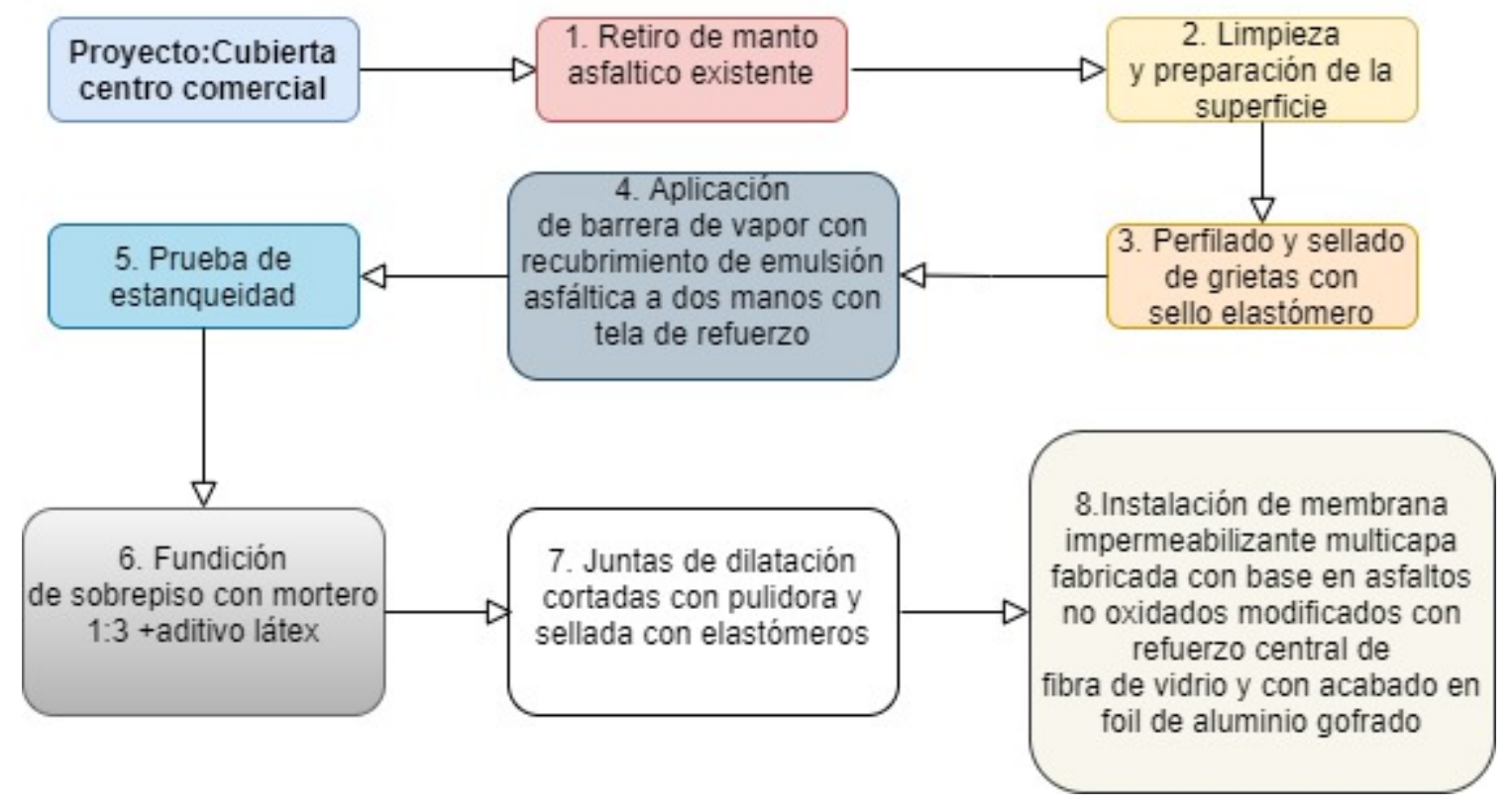

Figura 5: Flujograma proceso constructivo del proyecto de impermeabilización de cubierta de un centro comercial (elaboración Propia)

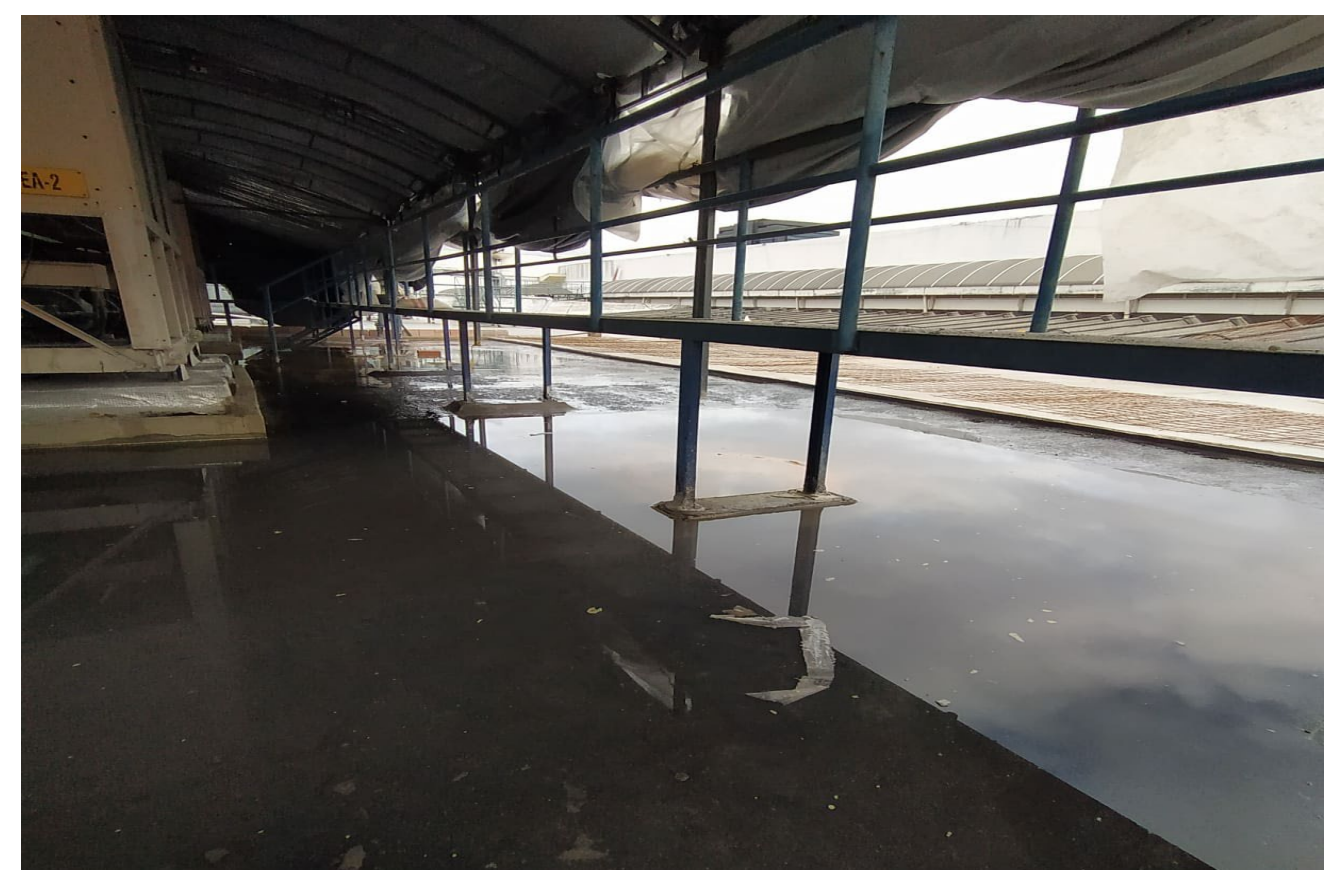

Figura 6: Prueba de estanqueidad. 


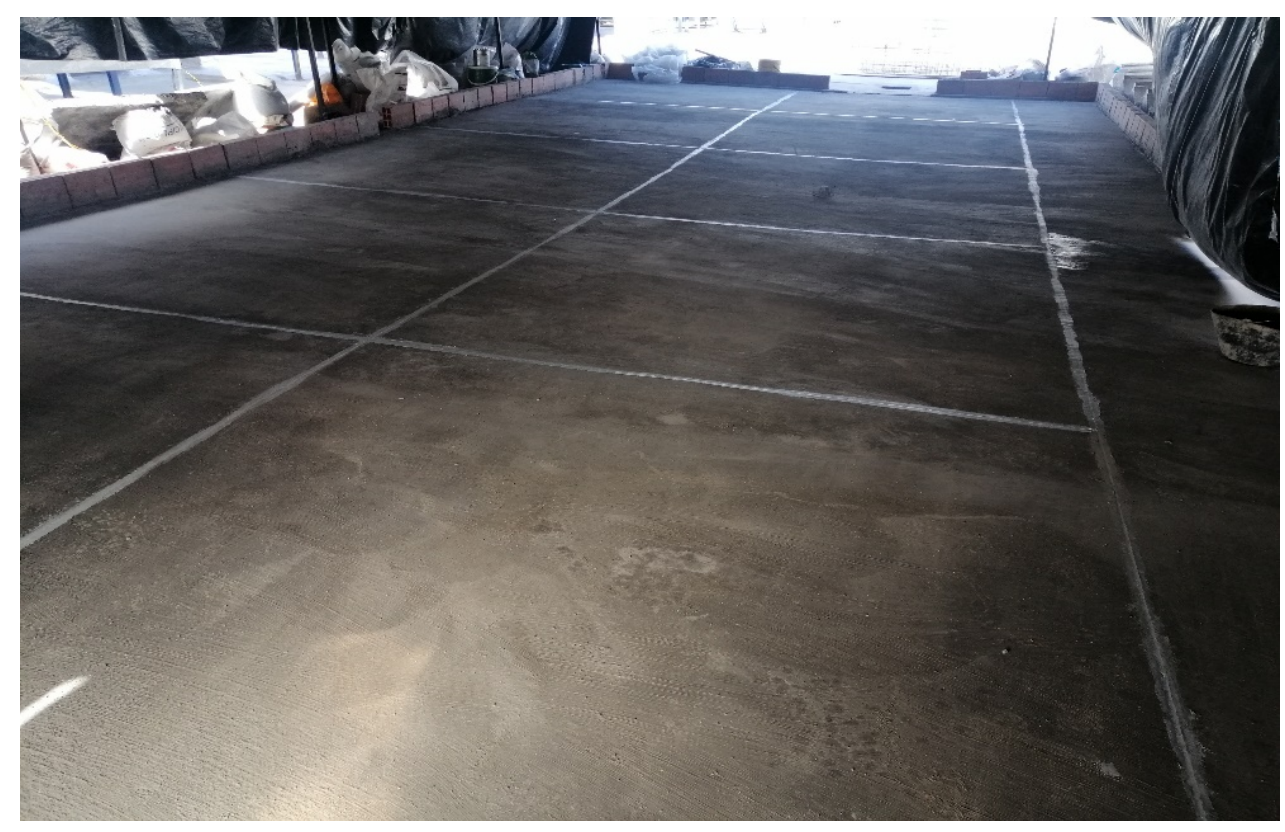

Figura 7: recubrimiento pesado sobre la impermeabilización con mortero 1:3 mejorado con aditivo látex y juntas de dilatación con sello elastómero para controlar cambios volumétricos por temperatura.

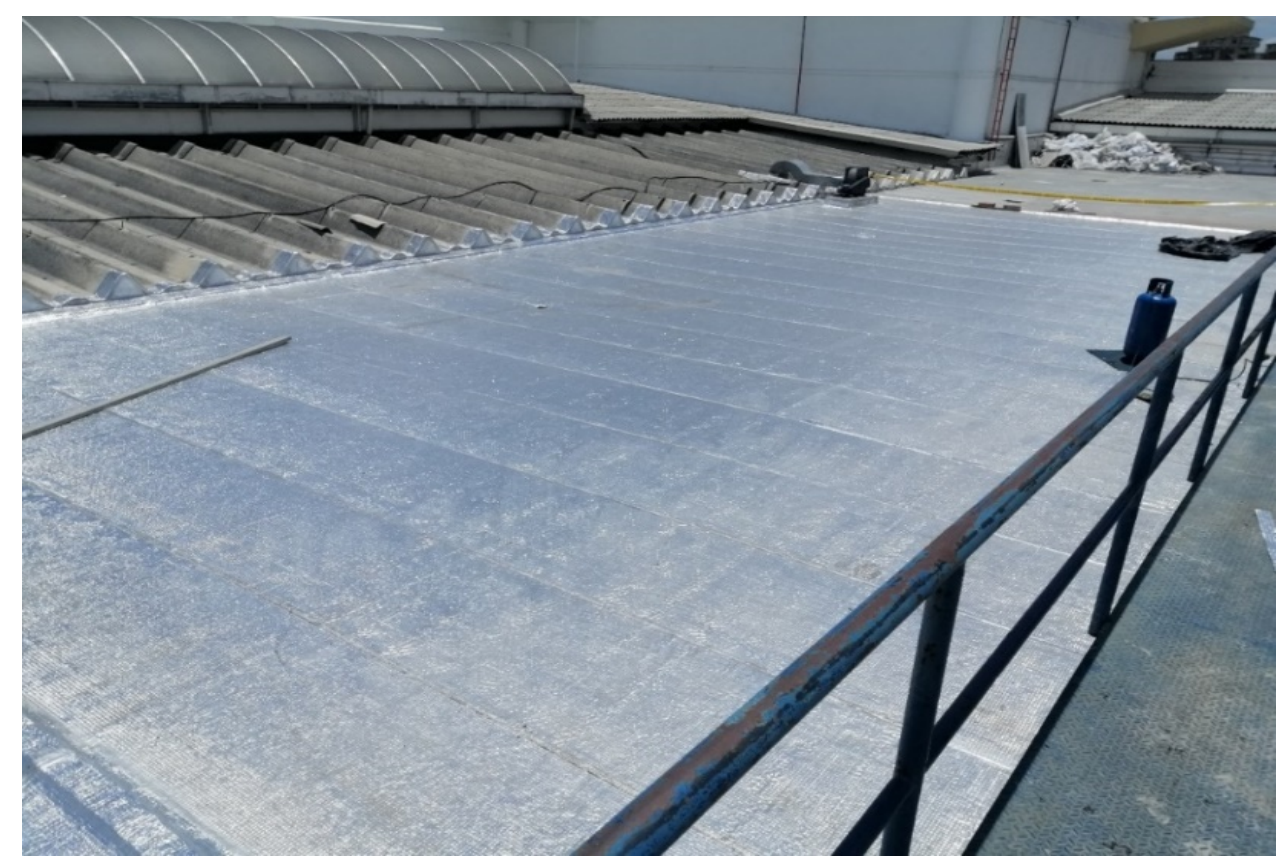

Figura 8: Recubrimiento de protección con manto multicapa fabricado con base en asfaltos no oxidados modificados, con refuerzo central de fibra de vidrio y con acabado en foil de aluminio gofrado.

Se comprueba por medio de la implementación del modelo en un caso práctico que al reducir la permeabilidad de una mezcla de concreto y aplicar un recubrimiento impermeable, considerando 
todas las etapas del proceso constructivo se puede llegar a una condición de estanqueidad en la cubierta plana de concreto reforzado y se reduce el riesgo de filtraciones que afecten el inmueble. Se entregó al personal de técnicos del mantenimiento in-house del cliente las recomendaciones de mantenimiento y su calendario. Sin embargo, es necesario realizar el seguimiento posterior a su entrega para garantizar que los procedimientos constructivos sean congruentes con el modelo propuesto.

\section{CONCLUSIONES}

- La impermeabilización es un proceso constructivo que requiere de un constante seguimiento desde la etapa de diseño hasta su entrega y posterior mantenimiento. El poder realizar el control en cada una de sus etapas garantizara que la vida útil del inmueble no se disminuya.

- El reducir la permeabilidad del concreto que constituye la cubierta es una solución que ayuda a disminuir el riesgo de filtraciones. Sin embargo, es un sistema colaborante y no es recomendable que sea la única opción para garantizar la estanqueidad. Por consiguiente, se debe colocar un recubrimiento impermeable sobre la superficie y a dicho recubrimiento $\mathrm{s}$ ele dará la mayor parte de la responsabilidad de la estanqueidad.

- Los criterios de aceptación en cualquier proyecto son necesarios para lograr que sea entregado a satisfacción. El plan de inspección de ensayos para proyectos de impermeabilización de cubiertas planas es una herramienta de gran valor que permitirá a los profesionales de la construcción evaluar el sistema de impermeabilización antes de su puesta en funcionamiento.

- El realizar el mantenimiento preventivo a los sistemas de impermeabilización de una cubierta plana en concreto permitirá alargar la vida útil del elemento de concreto reforzado. Si no se realiza el mantenimiento oportunamente la cubierta requerirá una rehabilitación antes del tiempo estimado.

- En general, el modelo propuesto es de gran pertinencia para la sociedad, porque permitirá optimizar el proceso de impermeabilización de cubiertas en concreto reforzado. Es de notar que la patología de la construcción es una disciplina que no solamente se orienta a reparar estructuras con lesiones, también puede enfocarse en mejorar el control de calidad del proceso constructivo antes de que se presenten daños o errores.

\section{AGRADECIMIENTOS}

Al grupo Toxement Euclid Group por el apoyo técnico y asesoría. A la firma ACOMEQ ingeniería SAS por permitir el uso de información de los proyectos de impermeabilización donde uno de los autores participó como líder de proyecto, los cuales fueron de gran utilidad para la investigación. A la Universidad Nacional de Colombia y sus facultades de artes y la facultad de ingeniería, brindaron apoyo a los autores en los temas que profundizan los diferentes campos de la construcción.

\section{REFERENCIAS}

- ACI Comite 201, (2012). Guía para la Durabilidad del Hormigón. Estados Unidos.

- AIS 100. (2010). TÍTULO B - Concreto estructural. NSR-10, TÍTULO C. Colombia. 
- AIS 100. (2010). TÍTULO C-Concreto estructural. NSR-10, TÍTULO C. Colombia.

- AIS 100. (2010). TÍTULO I - Supervisión técnica. NSR-10, TÍTULO I. Colombia.

- Alba-Cruz, R. C, Cruz-Álvarez, J.J, \& Posada, A.A (2013). "Mejora del proceso en el control de la calidad para el diseño de los sistemas de impermeabilización en las edificaciones". Revista de Arquitectura e Ingeniería, 7(2), 1-51. [fecha de Consulta 9 de Mayo de 2021]. ISSN: . Disponible en: https://www.redalyc.org/articulo.oa?id=193929227001

- Alba-Dorado, M. I. (2016). "Arquitectura y creatividad. Reflexiones acerca del proceso creativo del proyecto arquitectónico". Arquitectura revista, 12(2), 125-139. https://doi.org/10.4013/arq.2016.122.01

- Arredondo-Verdu, F. (1990). “Materiales de la construcción. Generalidades”. Dextra. Madrid. p.35.

- Carretero-Ayuso, M. J., \& de Brito, J. (2016). “Analysis of the Execution Deficiencies of Flat Roofs with Bituminous Membranes". Journal of Performance of Constructed Facilities. ASCE. 30(6), 18. https://doi.org/10.1061/(asce)cf.1943-5509.0000904

- Carretero, M. J., Moreno-Cansano, A., \& de Brito, J. (2017). Failure and damage determination of building roofs. Revista de La Construcción, 16(1), 145-157. https://doi.org/10.7764/RDLC.16.1.145

- Consorcio RehabiMed. (2007). "Método RehabiMed. Arquitectura Tradicional Mediterránea. II". Rehabilitación. Col·legi d'Aparelladors i Arquitectes Tècnics de Barcelona para el consorcio RehabiMed Bon Pastor. p.39-41.

- Cortés, J.M. (2017). "Sistema de gestión de calidad (ISO 9001:2001)”. ICB Editores-Bogotá: Ediciones de la U; 1ra edición. p. 178.

- Fernández, J. (2006). “Material Architecture”. (Vol. 21, Issue 1). Arquitectural Press \& Elsevier.

- García, L. E. (2014). "Desarrollo de la normativa sismo resistente colombiana en los 30 años desde su primera expedición”. Revista de Ingeniería Universidad de los Andes, 41, 71-77. https://doi.org/10.16924/riua.v0i41.785

- García-Ramírez, W. (2017). "A priori, A posteriori: la memoria descriptiva como reflexión teórica en arquitectura". Apuntes, 29 (1), 99-109. http://dx.doi.org/10.11144/Javeriana.apc29-1.mdrt

- Godinho-Lima, A.G. \& Verde-Zein, R. (2012). "Proyecto y Métodos proyectuales en La Investigación académica: algunos indicadores útiles". $4^{\text {a }}$ Jornadas Internacionales sobre Investigación en Arquitectura y Urbanismo, Valencia: (España), pp. 1-13.

- Gonçalves, M., Silvestre, J.D., de Brito, J., \& Gomes, R. (2019). "Environmental and economic comparison of the life cycle of waterproofing solutions for flat roofs". Journal of Building Engineering, 24(February), 100710. https://doi.org/10.1016/j.jobe.2019.02.002

- González-Domínguez, J., Sánchez-Barroso, G., \& García-Sanz-Salcedo, J. (2020). "Preventive maintenance optimisation of accessible flat roofs in healthcare centres using the Markov chain". Journal of Building Engineering. https://doi.org/10.1016/j.jobe.2020.101775

- Hernández-Moreno, S. (2008). "Teoría General de Sistemas aplicada al diseño arquitectónico sustentable". Revista Legado, 4(4), 55-66. https://legadodearquitecturaydiseno.uaemex.mx/article/view/13756

- Herrera-Cardenete, E, Martínez-Ramos, R. \& García-Nofuentes, J.F. (2016). "El proceso metodológico en el estudio de la patología de la construcción”. Opción, 32(9),918-928. [fecha de Consulta 9 de mayo de 2021]. ISSN: 1012-1587. Disponible en: https://www.redalyc.org/articulo.oa?id=31048482051

- ICONTEC. (1998). NTC 4483. ingeniería civil y arquitectura. Concretos. Métodos de ensayo para determinar la permeabilidad del concreto al água. Bogotá.

- ICONTEC. (2003). NTC 5222. Métodos de ensayo para medir el flujo libre, flujo restringido y segregación en concretos autocompactantes. Bogotá.

- ICONTEC. (2007). NTC 5551 Concretos. Durabilidad de estructuras de concreto. Ntc 5551,1-24. 
- ICONTEC. (2010). NTC 673. Concretos. Ensayo de resistencia a la compresión de especímenes cilíndricos de concreto. Bogotá.

- ICONTEC. (2015). NTC-ISO 9001. Sistemas de gestión de la calidad. Requisitos. Bogotá.

- ICONTEC. (2017). NTC 396. Concretos. Método de ensayo para determinar el asentamiento del concreto. Bogotá.

- ICONTEC. (2018). NTC 2871. Método de ensayo para determinar la resistencia del concreto a la flexión (utilizando una viga simple con carga en los tercios medios). Bogotá.

- INVIAS, (2013). I.N.V.E - 414-13. Resistencia a la flexión del concreto método de la viga simple cargada en los tercios de la luz. Bogotá.

- Lesur L. (2010), “Manual de Impermeabilización: una guía paso a paso”. (3rd Ed). Trillas. (original publicado en 1998).p 46-47.

- López-Rodríguez, F., Rodríguez-Rodríguez, V., Cruz-Astorqui, J.S, Torreño-Gómez, I, \& UbedaDe Mingo, P. (2004). Manual de patología de la edificación. Tomo 1 el lenguaje de las grietas patología y recalces de las cimentaciones. Universidad Politécnica de Madrid.

- Martínez-Osorio, P.A. (2013). "El proyecto arquitectónico como un problema de investigación". Revista de Arquitectura Universidad Católica, 15, 54-61. doi:10.14718/RevArq.2013.15.1.6

- Matallana-Rodríguez, R. (2019). "El concreto fundamentos y nuevas tecnologías”. Corona y Conconcreto. Bogotá.

- Medeiros, M.H.F., Real, L.V., Quarcioni, V.A, \& Helene, P. (2015). "Concreto con protección de superficie y expuesto a solución de cloruros: Espesor de recubrimiento equivalente". Revista ALCONPAT, 5(3), 209-223. https://doi.org/10.21041/ra.v5i3.90

- Monjo-Carrio, J. (1986). "Propuesta de evaluación de sistemas constructivos". Informes de La Construcción, 38(385), 5-29. https://doi.org/10.3989/ic.1986.v38.i385.1691

- Monjo-Carrio, J. \& Maldonado-Ramos, L. (2001). "Patología y técnicas de intervención en estructuras arquitectónicas". Munilla-Lería, Madrid.

- Monjo-Carrio, J. (2005). "La evolución de los sistemas constructivos en la edificación. Procedimientos para su industrialización. Informes de La Construcción", 57(499-500), 37-54. http://digital.csic.es/handle/10261/23065

- Montejo-Fonseca, A., Montejo-Piratova, F. \& Montejo-Piratova, A. (2013). "Tecnología y patología del concreto armado”. Universidad Católica de Colombia. Bogotá.

- Navarrete, S. (2016). "Enfoque fenomenológico de la crítica arquitectónica: el rol de la experiencia sensible”. Arquisur, 6 (9), 44-55. https://doi.org/10.14409/ar.v0i9.5802

- Osuna-Moral, J.J. (2016). "Pruebas de estanqueidad para cubiertas planas, identificación y solución de fallos en el desarrollo de las pruebas", Tesis Doctoral, Universidad de Alicante, p.29.

- Oteo-Mazo, C. (2018). “Patología geotécnica: Recalce y refuerzo del Terreno”. Grupo de proyectos de ingeniería E.T.S.I. Minas y Energía-Universidad Politécnica de Madrid; 1ra edición. p38.

- PMI. (2017). “Guía de los fundamentos para la dirección de proyectos PMBOK”. (6ta ed.). Estados unidos.

- Pedrosa, A., Del Río, M., \& Fonseca, C. (2014). “Interaction between plasticized polyvinyl chloride waterproofing membrane and extruded polystyrene board, in the inverted flat roof". Materiales de construcción, 64(316), 1-14. https://doi.org/10.3989/mc.2014.008913

- Peña-Beltrán, Z.Y. \& Pabón-Caicedo, J.D. (2020). "Climatology of hailstorms in Colombia". Cuadernos de Geografía: Revista Colombiana de geografía, 29(1), 259-282. https://doi.org/10.15446/rcdg.v29n1.75438

- Pillai, R.G., Gettu, R. \& Santhanam, M. (2020), "Uso de materiales cementicios suplementarios (SCM) en sistemas de concreto armado - Beneficios y limitaciones", Revista ALCONPAT, 10 (2), pp. 147 - 164, doi: https://doi.org/10.21041/ra.v10i2.477. 
- Racusin, R.G., "Building Deck Waterproofing from the Point of the Specifier's 'Pencil" Building Deck Waterproofing, ASTM STP 1084, L E. Gish, Ed.. American Society for Testing and Materials, Philadelphia, 1989, pp. 60-64. https://doi.org/10.1520/STP1084-EB

- Ruggiero, S.S. and Rutila, D.A., "Principles of Design and Installation of Building Deck Waterproofing," Building Deck Waterproofing, ASTM STP 1084, L. E. Gish, Ed., American Society for Testing and Materials, Philadelphia, 1990, 5-28. https://doi.org/10.1520/STP1084-EB

- Salas-Canevaro, J. D. (2017). La investigación en la actividad proyectual de la arquitectura. Objeto y método. 2(2), 5-15. https://doi.org/10.31381/pedagogiaarquitectura.v0i2.1299

- Wohl, R.L. and LaFraugh, R.W., "Criteria for the Selection of Penetrating Hydrophobic Sealers Used in the Repair of Concrete Parking Decks," Building Deck Waterproofing, ASTM STP 1084, L. E. Gish, Ed., American Society for Testing and Materials, Philadelphia, 1990, 75-82. https://doi.org/10.1520/STP1084-EB

- Villate, C. \& Tamayo, B. (2010). "La práctica de la arquitectura como racionalización sistémica". Revista de Arquitectura Universidad de los Andes, 1(6), 178-199. https://dialnet.unirioja.es/servlet/articulo? codigo $=3404962$ 